\title{
2D-Shape Analysis using Conformal Mapping
}

\author{
E. Sharon* \\ Division of Applied Mathematics \\ Brown University \\ Providence, RI 02912
}

\author{
D. Mumford* \\ Division of Applied Mathematics \\ Brown University \\ Providence, RI 02912
}

\begin{abstract}
The study of 2D shapes and their similarities is a central problem in the field of vision. It arises in particular from the task of classifying and recognizing objects from their observed silhouette. Defining natural distances between $2 D$ shapes creates a metric space of shapes, whose mathematical structure is inherently relevant to the classification task. One intriguing metric space comes from using conformal mappings of $2 D$ shapes into each other, via the theory of Teichmüller spaces. In this space every simple closed curve in the plane ( $a$ "shape") is represented by a 'fingerprint' which is a diffeomorphism of the unit circle to itself (a differentiable and invertible, periodic function). More precisely, every shape defines to a unique equivalence class of such diffeomorphisms up to right multiplication by a Möbius map. The fingerprint does not change if the shape is varied by translations and scaling and any such equivalence class comes from some shape. This coset space, equipped with the infinitesimal Weil-Petersson (WP) Riemannian norm is a metric space. In this space, it appears very likely to be true that the shortest path between each two shapes is unique, and is given by a geodesic connecting them. Their distance from each other is given by integrating the WP-norm along that geodesic. In this paper we concentrate on solving the "welding" problem of "sewing" together conformally the interior and exterior of the unit circle, glued on the unit circle by a given diffeomorphism, to obtain the unique 2D shape associated with this diffeomorphism. This will allow us to go back and forth between $2 D$ shapes and their representing diffeomorphisms in this "space of shapes".
\end{abstract}

\section{Introduction}

Many different representations for the collection of all $2 D$ shapes, and many different measures of similarity between them have been studied recently $[15,16,22,1,23,20$, $17,12,13,11,2,3,21,7,10,10,14]$. Although significant progress has been made, none are satisfactory from the

\footnotetext{
* Research was supported by NSF grant DMS-0074276.
}

point of view of a leading to a successful classification of the collection of all shapes. In part, this shortcoming is due to the fact that we as humans may give different meanings to similarity between shapes in different contexts [4, 19]. In this paper, we propose the study of a new approach to the collection of all shapes by applying the mathematical theory of complex analysis.

We begin a mathematical analysis of the set of all simple closed curves in the plane up to translations and scalings ("shapes"), by embedding these shapes as points in a metric space that faithfully represents their continuous variability, and that allows their classification by similarity (i.e. the inverse of their metric distances). Note that there is no natural linear structure to the set of shapes, and therefore it is undesirable to simply map this set to $\Re^{n}$, say, by characterizing each shape using some $n \in \mathcal{N}$ real-valued features (e.g. [5]). In addition, it is obvious that the space of shapes has to be infinite dimensional to represent all the variability of all possible simple closed curves. Therefore, embedding the set of shapes in simplistic spaces, like $\Re^{n}$ may easily result in wrong shape classifications.

Many metric approaches for the classification of shapes have been suggested. Typically, a pairwise distance between shapes is set up by some $2 D$ spatial comparison between the two closed curves (e.g. the Hausdorff distance). Then a generic clustering algorithm is employed for dividing some database of shapes into categories (see [9]). This approach is more accommodating than simply embedding the shapes as if they were points in $\Re^{n}$, but still lacks the appropriate structure for morphing between shapes for example. It is also a challenge to find a meaningful pairwise distance on one hand, and an appropriate clustering algorithm on the other hand.

In our space of shapes, every shape will be represented by a diffeomorphism of the unit circle to itself, that is a smooth function $f: \Re \rightarrow \Re$ which is differentiable, invertible and satisfies $f(x+2 \pi)=f(x)+2 \pi$. (Equivalently, every such function can be viewed as a real-valued function from $[0,2 \pi]$ to itself.) More precisely, we will show that every simple closed curve in the plane, up to scaling and translation, corresponds to an equivalence class of such diffeomorphisms. These equivalence classes are 
the right cosets of these diffeomorphisms modulo the three dimensional subgroup of Möbius maps, namely the maps from the complex unit circle $\{z|| z \mid=1\}$ to itself given by $z \mapsto(a z+b) /(\bar{b} z+\bar{a})$. This quotient space equipped with the Weil-Petersson Riemannian norm is a metric space.

The construction is based on the theory of conformal mappings of $2 \mathrm{D}$ regions into each other, via the theory of Teichmüller spaces. Conformal mappings are of great interest since they preserve the angles between any two intersecting curves when mapping between differently-shaped regions, and are unique up to a Möbius-transformation ambiguity - which is factored out when using the quotient space of diffeomorphisms. The resulting space has two major properties. First, the space has non-positive sectional curvature [6], which, if some completeness properties hold, implies that there exists a unique geodesic between each two shapes. Defining the integral of the WP-norm along a path as the length of this path, a geodesic is defined as the shortest path connecting the two shapes and its length is the global metric on the space of shapes. Moreover, the shapes along that path represent a natural morphing of one into the other. Secondly, the resulting space is homogenous with respect to the group of diffeomorphisms operating on the cosets from the left. Thus, for example, we can transform all shapes into new shapes by composing the coset representing them by a diffeomorphism on the left and this transformation will preserve distance change the above morphing between any two shapes into the morphing between the transformed shapes.

It is essential in this framework to be able to move back and forth between $2 D$ shapes and the diffeomorphisms representing them. Moving from a given shape into the diffeomorphism representing it is not so hard and, for example, can be simply solved by using an existing numerical implementation of the Schwarz-Christoffel formula, applied to a polygon that tightly approximates the shape. But going back from the diffeomorphism to the shape is a major computational challenge, known as the "welding" problem. It involves the construction of two conformal maps, one defined inside the unit circle and one outside, which differ on the unit circle by the given diffeomorphism. Having this transformation between the space of shapes and the group of diffeomorphisms will also allow us next to illustrate the morphing of one shape into the other, along the shortest path connecting them. For this, we will have to compute the geodesics in the quotient space of diffeomorphisms, as we intend to do in a subsequent paper.

In this paper we concentrate on representing the $2 D$ shapes in the space we suggest, demonstrating how to move back and forth between the two presentations of $2 D$ shapes: a simple, closed curve and a representative diffeomorphism from its corresponding coset in the quotient space. Note that this means that every shape, up to scaling and transla- tion, can be naturally described by a "fingerprint", which is a diffeomorphism of the circle - a $1 D$, real-valued periodic function of $[0,2 \pi]$ to itself.

\section{Shapes as diffeomorphisms of the circle via conformal mappings}

In this paper, by a "shape" we mean a simple closed smooth curve in the plane. Smooth means having derivatives of all orders (i.e. being $C^{\infty}$ ), and simple means that the curves that do not intersect themselves. For this we make use of Riemann's conformal mapping theorem which states that it is possible to map the unit disc conformally to the interior of any other such shape. The conformal transformation is unique up to any preceding Möbius transformations mapping the unit disc to itself (that is, maps of the form $z \mapsto(a+b) /(\bar{b} z+\bar{a}))$. Conformal means that the infinitesimal angle between each two crossing curves is equal to the infinitesimal angle between the transformed curves. This means for instance, that if we place a radial grid on the unit disc, made out of concentric circles and lines through the origin, the right angles between the lines and circles are going to be preserved in the transformed grid, projected on the transformed shape.

\subsection{Shapes to diffeomorphisms}

We associate $\Re^{2}$ with the complex plane $\mathcal{C}$, we denote planar points by complex numbers $u+i v$, denote the unit disc by $\Delta_{-}$and the infinite region outside the unit disc plus the unit circle by $\Delta_{+}$. For every simple closed curve $\Gamma$ in $\mathcal{C}$ we denote by $\Gamma_{-}$its union with the region enclosed by it, and denote by $\Gamma_{+}$its union with the infinite region outside $\Gamma$. Then by the Riemann mapping theorem, for all $\Gamma$ there exists a conformal map $\Phi_{-}: \Delta_{-} \rightarrow \Gamma_{-}$, unique up to replacing $\Phi_{-}$by $\Phi_{-} \circ A$ for any Möbius transformations $A: \Delta_{-} \rightarrow \Delta_{-}, A=\frac{a z+b}{b z+\bar{a}}$. That is, for every two conformal maps $\Phi_{-}^{(1)}, \Phi_{-}^{(2)}: \Delta_{-} \rightarrow \Gamma_{-}$we have that $\Phi_{-}^{(2)^{-1}} \circ \Phi_{-}^{(1)}=A$, where $A: \Delta_{-} \rightarrow \Delta_{-}$is a conformal, Möbius map from the unit disc to itself as defined above.

We denote the extended complex plane or Riemann sphere by $\hat{\mathcal{C}} \doteq \mathcal{C} \cup\{\infty\}$, and add the point at infinity to $\Delta_{+}$ and $\Gamma_{+}$. We can think of $\Gamma_{-}$and $\Gamma_{+}$as a partition of the Riemann sphere into two parts along $\Gamma$. Observe that using the transformation $1 / z$ we can identify $\Delta_{+}$with $\Delta_{-}$. Moreover, under $1 / z, \Gamma$ is transformed into the inverted simple closed curve $\Gamma^{\prime}$ so that $\Gamma_{+}$is identified with the interior $\Gamma_{-}^{\prime}$ of $\Gamma^{\prime}$. Thus we can apply the Riemann mapping theorem to get a $\Phi^{\prime}$ from $\Delta_{-}$and $\Gamma_{-}^{\prime}$. Composing this conformal map with inverse on both sides, i.e. $\Phi_{+}(z)=1 / \Phi^{\prime}(1 / z)$, we get a conformal map of the exteriors $\Phi_{+}: \Delta_{+} \rightarrow \Gamma_{+} \cdot \Phi_{+}$ is also unique up to Möbius transformations as above. But now we can also require that $\Phi_{+}$carries $\infty$ to $\infty$, and that 
its differential carries the real positive axis of the $\Delta$-plane at $\infty$ to the real positive axis of the $\Gamma$-plane at $\infty$. Thus, we eliminate the Möbius ambiguity of $\Phi_{+}$for every $\Gamma$, and make it unique.

The goal of this construction is the conformal map $\Psi \doteq$ $\Phi_{+}{ }^{-1} \circ \Phi_{-}$, on the domain on which it is defined, namely the unit circle $S^{1}$. (Note that $\Phi_{-}\left(S^{1}\right)=\Gamma$, and $\Phi_{+}{ }^{-1}(\Gamma)=$ $S^{1}$.) In fact, $\Psi: S^{1} \rightarrow S^{1}$ is a diffeomorphism, which can be thought of as a periodic, real-valued function from $[0,2 \pi]$ to $[0,2 \pi]$, having a positive derivative everywhere. $\Psi$ is a uniquely-identifying fingerprint of the shape $\Gamma$.

\subsection{Shapes as equivalence classes of diffeo- morphisms}

From the Möbius-transformation ambiguity left in $\Phi_{-}$we can see that by the construction of $\Psi$ every simple closed curve $\Gamma$ induces a diffeomorphism $\Psi: S^{1} \rightarrow S^{1}$, which is unique up to preceding it by a diffeomorphism $\tilde{A}: S^{1} \rightarrow$ $S^{1}$ coming from the restriction to $S^{1}$ of any Möbius transformation $A: \Delta_{-} \rightarrow \Delta_{-}$. We denote the group of all diffeomorphisms $\Psi: S^{1} \rightarrow S^{1}$ by $\operatorname{Diff}\left(S^{1}\right)$, and the subgroup of all the restricted Möbius transformations, $\tilde{A}: S^{1} \rightarrow S^{1}$ by $\mathbf{P S L}_{2}(\Re)$.

We denote our quotient space by $G / H$, where $G=$ $\operatorname{Diff}\left(S^{1}\right)$ and $H=\mathbf{P S L}_{2}(\Re)$. An element in $G / H$ is determined by $g \in G$, and will be referred to as the coset $g H \doteq\{g \circ h \mid h \in H\}$. We denote the space of simple closed smooth curves $\Gamma$ by $\mathcal{S}$, and by $\overline{\mathcal{S}}$ the same space modulo scaling and translation of its curves. By our construction of $\Psi$ in Sec. 2.1, we get a map from $\overline{\mathcal{S}}$ to $G / H$. Remarkably, this map is a bijection, meaning that for every coset $g H$ in $G / H$, there exists a unique $\bar{\Gamma} \in \overline{\mathcal{S}}$ such that any $\Gamma$ representing $\bar{\Gamma}$ (same $2 D$ shape up to scale and translation) will generate a $\Psi$ in $g H$. Thus we have that

$$
G / H \cong \overline{\mathcal{S}} .
$$

\subsection{Diffeomorphisms to shapes: welding}

To obtain the unique $\Gamma$ corresponding to any such coset $g H \in G / H$ we may pick any $\Psi$ in $g H$. We may construct an abstract Riemann surface by welding $\Delta_{+}$and $\Delta_{-}$ using the map $\Psi$ to identify their boundaries and apply the classification of simply connected Riemann surfaces to say that the result must be the same as $\hat{\mathcal{C}}$. The usual way to do this is to cite Bers' theorem [18] which gives us a curve $\Gamma$, and two conformal mappings $\Phi_{-}: \Delta_{-} \rightarrow \Gamma_{-}$and $\Phi_{+}: \Delta_{+} \rightarrow \Gamma_{+}$, such that $\left.\Phi_{+}^{-1} \circ \Phi_{-}\right|_{S^{1}}=\Psi$. The objects $\left\{\Gamma, \Phi_{-}, \Phi_{+}\right\}$are unique up to replacing them by $\left\{A(\Gamma), A \circ \Phi_{-}, A \circ \Phi_{+}\right\}$with an arbitrary Möbius transformation $A(z)=(a z+b) /(c z+d)$ (now not necessarily mapping the unit circle to itself). We may also require that $\Phi_{+}(\infty)=\infty$ and that the derivative of $\Phi_{+}$at $\infty$ map the tangent to the positive real axis to itself. This makes $\left\{\Gamma, \Phi_{-}, \Phi_{+}\right\}$unique up to changing them as above with $A(z)=a z+b, a>0$ real. Thus the constructed $\Gamma$ is unique up to translations and scalings as required.

\subsection{The homogeneous structure of $\overline{\mathcal{S}}$}

The group $G$ operates, of course, on the coset space $G / H$, hence, as a result of the above construction, it operates also on the space of shapes $\overline{\mathcal{S}}$. A concrete way of defining this action is this: to transform any $\bar{\Gamma} \in \bar{S}$ by a group element $\Psi$, we construct the conformal map $\Phi_{+}: \Delta_{+} \rightarrow \Gamma_{+}$hence we get the map $\Psi^{\prime}=\Phi_{+} \circ \Psi \circ \Phi_{+}^{-1}$ from $\Gamma$ to itself. Then we use the same welding trick by cutting open $\hat{\mathcal{C}}$ along $\Gamma$ and rewelding it with the map $\Psi^{\prime}$. Applying Bers' theorem, the result can be conformally mapped to the extended sphere, taking $\Gamma$ to a new curve $\Gamma^{\prime}$. This way we get a transitive group operation on $\overline{\mathcal{S}}$.

\section{The WP Riemannian metric on $\overline{\mathcal{S}}$}

\subsection{The WP norm on the Lie algebra of $G$}

The Lie algebra of the group $G$ is given by the vector space of smooth vector fields on the circle: $\phi(\theta) \partial / \partial \theta$ where $\phi(\theta+$ $2 \pi)=\phi(\theta)$. The adjoint action of $g \in G$ is the linear map from $\operatorname{Lie}(G)$ to itself induced by the conjugation map $h \mapsto g^{-1} \circ h \circ g$ from $G$ to itself. Explicitly, this maps $\phi \in \operatorname{Lie}(G)$ to $\phi \circ g / g^{\prime}$, i.e. $a d_{g}(\phi)=\phi \circ g / g^{\prime}$.

We can expand such a $\phi$ in a Fourier series $\phi(\theta)=$ $\sum_{n=-\infty}^{\infty} a_{n} e^{i n \theta}$ (where $\overline{a_{n}}=a_{-n}$ ). The Weil-Petersson norm on $\operatorname{Lie}(G)$ is defined by:

$$
\|\phi\|_{W P}^{2}=\sum_{n=2}^{\infty}\left(n^{3}-n\right)\left|a_{n}\right|^{2} .
$$

The null space of this norm is given by those vector fields whose only Fourier coefficients are $a_{-1}, a_{0}$ and $a_{1}$, i.e. the vector fields $(a+b \cos (\theta)+c \sin (\theta)) \partial / \partial \theta$, which are exactly those tangent to the Möbius subgroup $H$, i.e. in the Lie algebra of $H$.

The motivation for this particular definition is the fact that, for all $h \in H$ and $\phi \in \operatorname{Lie}(G)$, one can verify that

$$
\left\|a d_{h}(\phi)\right\|_{W P}=\|\phi\|_{W P} .
$$

\subsection{Extending the metric to $G / H$}

A general fact on metrics on coset spaces is that a Riemannian metric on $G / H$ which is invariant by all left multiplication maps $L_{g}: G / H \rightarrow G / H$, for all $g \in G$ is given by a norm $\|\phi\|$ on the Lie algebra of $G$ which is zero on the Lie subalgebra of $H$ and which satisfies $\left\|a d_{h}(\phi)\right\|=\|\phi\|$ for all $h \in H$. Here the norm on the tangent space $T_{g H, G / H}$ to 
$G / H$ at any $g H$ is gotten from the norm on the Lie algebra via the isomorphism

$$
D L_{g}: \operatorname{Lie}(G) / \operatorname{Lie}(H)=T_{e H, G / H} \rightarrow T_{g H}
$$

given by the derivative of $L_{g}$ at the identity $e$ of $G$. In particular, this applies to our $G$ and $H$. Because $G / H \cong \overline{\mathcal{S}}$, we have now constructed a homogeneous Riemannian metric on $\overline{\mathcal{S}}$ also.

Next let's translate this into concrete terms. Take any path $\Psi(t, \theta)$ in $G$, where $t \in\left[0, t_{0}\right] \subset \Re$ and $\Psi(t, \theta+2 \pi)=$ $\Psi(t, \theta)+2 \pi$. The tangent vectors to this path are given by $\frac{\partial \Psi(t, \theta)}{\partial t}=\Psi_{t}(t, \theta)$ or, translated back to the Lie algebra by left multiplication by $\Psi^{-1}$, by $\Psi_{t}(t, \theta) / \Psi_{\theta}(t, \theta)$. We expand the tangent vector at every $t \geq 0$ by its Fourier series in $\theta$ :

$$
\Psi_{t}(t, \theta) / \Psi_{\theta}(t, \theta)=\sum_{n=-\infty}^{\infty} a_{n}(t) e^{i n \theta}
$$

where $a_{-n}(t)=\overline{a_{n}(t)}$ because the vector field is real. Its Weil-Petersson norm is then given by

$$
\left\|\Psi_{t}(t, \theta) / \Psi_{\theta}(t, \theta)\right\|_{\mathrm{W} P} \doteq \sum_{n=2}^{\infty}\left|a_{n}(t)\right|^{2}\left(n^{3}-n\right)
$$

and the length of the path is by definition: $\int_{0}^{t_{0}} \sqrt{\sum_{n=2}^{\infty}\left|a_{n}(t)\right|^{2}\left(n^{3}-n\right)} d t$.

\section{Computing shapes from diffeomor- phisms and vice versa}

\subsection{Schwarz-Christoffel: shapes to diffeo- morphisms}

Arguably the most computationally efficient way to compute the conformal map $\Phi$ from the unit disk to the interior of a simple closed curve $\Gamma$ is to approximate $\Gamma$ by a polygon and use the Schwarz-Christoffel formula. This formula is as follows: let $z$ be the complex coordinate in the unit disk, let $\left\{a_{i}\right\}$ be the points on the unit circle which will map to the vertices of the polygon and let $\left\{\pi \alpha_{i}\right\}$ be the angles of the polygon at these vertices. Then for some $C_{1}, C_{2}$ :

$$
\Phi(z)=C_{1} \int^{z} \prod_{i}\left(z-a_{i}\right)^{\alpha_{i}-1} d z+C_{2} .
$$

This method has been implemented in the excellent package 'sc' by Tobin Driscoll, based on earlier work by L.N. Trefethen, and distributed at www.math.udel.edu/ driscoll/SC [8]. The key problem is that one is usually given only the points $\Phi\left(a_{i}\right)$ and must compute $\left\{a_{i}\right\}$ at the same time as $\Phi$. Moreover, they are non-unique as, for any Möbius map $A$, $\Phi \circ A,\left\{A^{-1}\left(a_{i}\right)\right\}$ are equally good solutions. The program allows you to specify the point $\Phi(0) \in \operatorname{Int}(\Gamma)$ to get the best looking and best behaved solution. (See Fig. 1 for an example.)

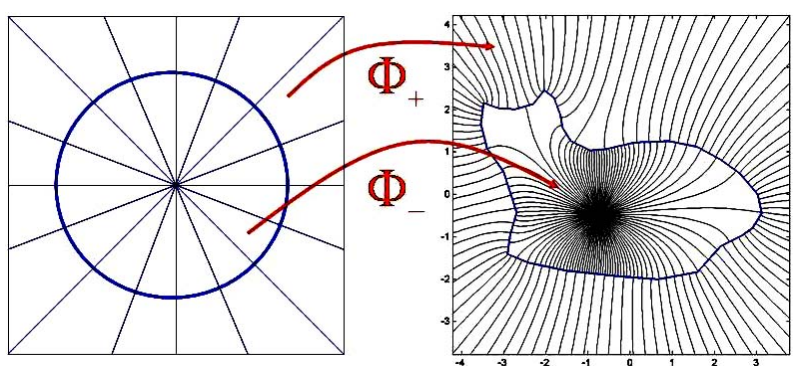

Figure 1: Using Schwarz-Christoffel. The conformal mappings $\Phi_{-}$and $\Phi_{+}$, described in Sec. 2.1, carry a homogenous radial grid (left, drawn schematically) onto the interior and exterior of $\Gamma$ separately (right).

\subsection{Welding: diffeomorphisms to shapes}

\subsubsection{Setting up the analytic welding equations}

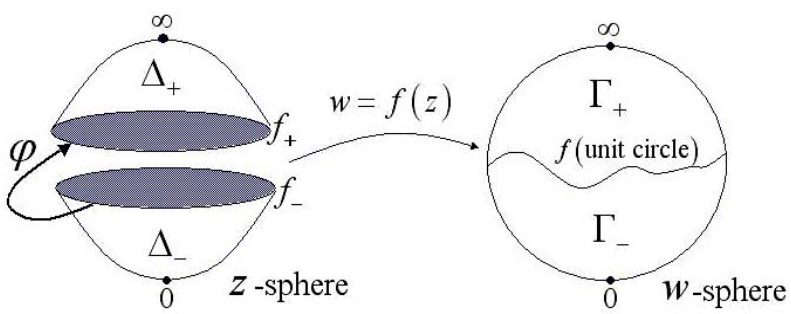

Figure 2: The conformal map $f$, as described in Sec.4.2.1, maps the two halves of the $z$-sphere divided by the unit circle (left) onto the two parts of the $w$-sphere divided by $\Gamma$ (right), correspondingly, such that $f_{-}(z)=$ $f_{+}(\varphi(z))$ on $|z|=1$.

We consider $\Delta_{-}$and $\Delta+$ as a partition of the Riemann sphere into two parts along the unit circle $\Delta$, and $\Gamma_{-}$and $\Gamma_{+}$as a partition of the Riemann sphere into two parts along $\Gamma$, as explained in Sec. 2.1 (see Fig. 2). We associate the complex-plane variable $z$ with the $\Delta$-sphere, and the complex-plane variable $w$ with the $\Gamma$-sphere. We will assume that $0 \in \Delta_{-}$in order to normalize the map $\Phi_{-}$as well as $\Phi_{+}$by asking that $\Phi_{-}(0)=0$. Given a diffeomorphism $\varphi: \Delta \rightarrow \Delta$, we seek a function $f(z)$ from the $z$-sphere minus the unit circle to the $w$-sphere, complex analytic on $|z|<1$ with boundary values $\left.f\right|_{|z|=1}=f_{-}$, and on $|z|>1$ with $\left.f\right|_{|z|=1}=f_{+}$, such that

$$
\begin{aligned}
& f(0)=0, \quad f(\infty)=\infty \\
& f_{-}(z)=f_{+}(\varphi(z)) \quad|z|=1
\end{aligned}
$$

Note that $f(z) / z$ has finite non-zero limiting values at 0 and $\infty$, hence it has a single-valued logarithm in $\Delta_{-}$and $\Delta_{+}$. Thus we may define $g(u)$ by

$$
\log \left(\frac{f\left(e^{u}\right)}{e^{u}}\right)=g(u), u \in \widehat{\mathcal{C}}-S^{1}
$$


so that $g(u+2 \pi i) \equiv g(u)$.

Now,

$$
\begin{array}{ll}
\operatorname{Re}(u) \rightarrow-\infty & \Rightarrow\left|e^{u}\right| \rightarrow 0 \\
\Rightarrow f\left(e^{u}\right) \approx c_{1} e^{u} & \Rightarrow g(u) \approx \log c_{1},
\end{array}
$$

and

$$
\begin{array}{ll}
\operatorname{Re}(u) \rightarrow \infty & \Rightarrow\left|e^{u}\right| \rightarrow \infty \\
\Rightarrow f\left(e^{u}\right) \approx c_{2} e^{u} & \Rightarrow g(u) \approx \log c_{2},
\end{array}
$$

for some constants $c_{1}$ and $c_{2}$. Without loss of generality, we can replace $f$ by $c_{2}^{-1} f$ so that $c_{2}=1$, and $g(u) \approx 0$ as $\operatorname{Re}(u) \rightarrow+\infty$.

We define $\Psi: \Re \rightarrow \Re$, satisfying $\Psi(\theta+2 \pi)=\Psi(\theta)+2 \pi$ by $\varphi\left(e^{i \theta}\right)=e^{i \Psi(\theta)}$. Then,

$$
\begin{aligned}
& g_{-}(i \theta)=\log \left(f_{-}\left(e^{i \theta}\right)\right)-i \theta \\
& \begin{aligned}
g_{-}(i \theta) & =\log \left(f_{-}\left(e^{i \theta}\right)\right)-i \theta \\
\log \left(f_{+}\left(\varphi\left(e^{i \theta}\right)\right)\right)-i \theta & =\log \left(f_{+}\left(e^{i \Psi(\theta)}\right)-i \theta\right.
\end{aligned} \\
& =g_{+}(i \Psi(\theta))+i \Psi(\theta)-i \theta \text {. }
\end{aligned}
$$

Thus we get a new welding condition on the imaginary axis

$$
g_{-}(i \theta)=g_{+}(i \Psi(\theta))+i(\Psi(\theta)-\theta) .
$$

Note that if Eq. 9 holds at $\theta$ then it also holds at $\theta+2 \pi$.

Now let

$$
g(u)=h(u)+i k(u)
$$

where $h, k$ are real. Then,

$$
\begin{array}{lll}
h, k \text { harmonic } & \text { if } & \operatorname{Re}(u)<0, \operatorname{Re}(u)>0 \\
h, k \rightarrow 0 & \text { if } \operatorname{Re}(u) \rightarrow+\infty \\
h, k \rightarrow \text { suitable constants } & \text { if } \operatorname{Re}(u) \rightarrow-\infty \\
h, k \text { periodic } & \text { if } u \rightarrow u+2 \pi i .
\end{array}
$$

Furthermore, from Eq. 9 we get that

$$
\begin{aligned}
& h_{-}(i \theta)=h_{+}(i \Psi(\theta)) \\
& k_{-}(i \theta)=k_{+}(i \Psi(\theta))+\Psi(\theta)-\theta .
\end{aligned}
$$

By the Cauchy-Riemann equations, if $u=s+i \theta$, we have for $s<0, s>0$ that

$$
\frac{\partial h}{\partial \theta}=-\frac{\partial k}{\partial s}, \quad \frac{\partial h}{\partial s}=\frac{\partial k}{\partial \theta} .
$$

For $s=0$ this gives

$$
-\frac{\partial k_{-}}{\partial s}=\frac{\partial h_{-}}{\partial \theta}=\Psi^{\prime}(\theta) \frac{\partial h_{+}}{\partial \theta}=-\Psi^{\prime}(\theta) \frac{\partial k_{+}}{\partial s} .
$$

Thus, we can conclude the following conditions on $k$

$$
\begin{array}{ll}
k \text { harmonic on } s<0, s>0 & \\
k \text { periodic w.r.t. } \theta \rightarrow \theta+2 \pi & \\
k \rightarrow 0 \text { if } s \rightarrow \infty, k \rightarrow \mathrm{c} \text { if } s \rightarrow-\infty & \\
\left.k_{-}\right|_{i \theta}=\left.k_{+}\right|_{i \Psi(\theta)}+\Psi(\theta)-\theta & \text { on } s=0 \\
\left.\frac{\partial k_{-}}{\partial s}\right|_{i \theta}=\left.\Psi^{\prime}(\theta) \frac{\partial k_{+}}{\partial s}\right|_{i \Psi(\theta)} & \text { on } s=0,
\end{array}
$$

for some real constant $c$ which comes implicitly from the equations. Note that Eq. 15 is in fact an equation for a real function $k$, of the two real variables $s$ and $\theta$. Having solved it for $k=k(s, \theta)$ we get $h=h(s, \theta)$ as the conjugate function of $k$, via the Cauchy-Riemann relations in Eq. 13. From Eq. 5 and Eq. 10 we get that

$$
f\left(e^{s+i \theta}\right)=e^{h(s+i \theta)+s+i(k(s+i \theta)+\theta)} .
$$

Since $\Gamma$ is given by $\left.f(\theta)\right|_{s= \pm 0}$, we have that $\left.h(\theta)\right|_{s= \pm 0}$ and $\left.k(\theta)\right|_{s= \pm 0}$ describe the the magnitude and angle, respectively, of the complex-plane vectors delineating $\Gamma$ as a periodic function of $\theta$.

\subsubsection{Solving the welding equations numerically}

Given a diffeomorphism $\Psi$, we solve Eq. 15 for the $\theta$ periodic function $k=k(s, \theta)$ on the plane branch $\theta \in$ $[0,2 \pi]$ and $-\infty<s<\infty$. We conveniently set three different, staggered grids on $(s, \theta)$, with uniform meshsize $h>0$ for the three functions $k, h$ and $g$ (see the square inset in Fig. 3). In practice, we cut off the $s$ direction into $-\hat{s} \leq s \leq \hat{s}$, for some $\hat{s}>0$, at which the values of $k$, $h$ and $g$ already converge to constants (cf. Eq. 11). Solving for $k$ on the $k$-grid, we use Eq. 13 to compute $h$ on the $h$-grid, and interpolating both to get $g$ on the $g$-grid. It is the values of $g$ on $s= \pm 0$ that fix the resulting curve $\Gamma$. In practice, having $k$, we directly compute $h$ on $s= \pm 0$, at the $k$-grid points, as explained at the end of Sec. 4.2.2.

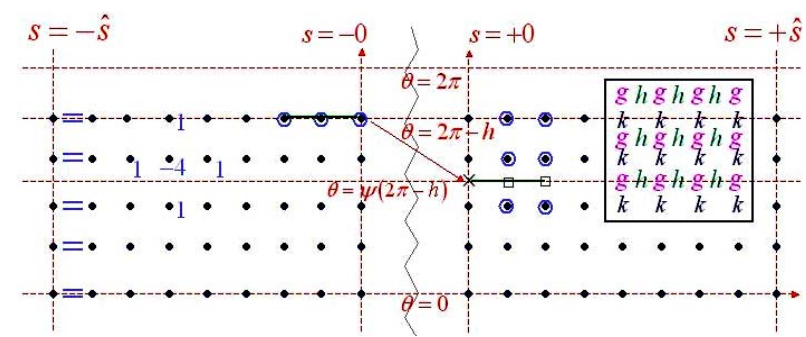

Figure 3: The $\left(s_{i}, \theta_{j}\right) k$-grid on the $(s, \theta)$ plane. In blue over the grid points, a schematic sketch of the three types of equations involved in the numeric solution of $k$, as described in Sec. 4.2.2: the zero derivatives at the external boundaries $(s= \pm \hat{s}$ ), described by the equal signs (Neuman boundary conditions), the template of the Laplacian mask applied to internal grid points $(s \neq \pm 0, \pm \hat{s})$, and the 9 grid points (circled) involved in the internal boundary condition for every internal-boundary grid point $(s= \pm 0)$. The square inset demonstrates the three staggered grids, for the functions $k, h$ and $g$. Every grid point is represented by the corresponding letter.

Solving for $k(s, \theta)$, we define the $\left(s_{i}, \theta_{j}\right) k$-grid, by indexing with $i, j \in \mathcal{N}$ an $s$-grid: $s_{i} \in\{-\hat{s},(-\hat{s}+h),(-\hat{s}+$ $2 h), \ldots,-2 h,-h,-0,+0, h, 2 h, \ldots,(\hat{s}-2 h),(\hat{s}-h), \hat{s}\}$, and a $\theta$-grid: $\theta_{j} \in\{0, h, 2 h, \ldots,(2 \pi-h)\}$ (for which the index $j$ applies periodically). We set three different types of equations.

First we have the basic simplest second-order discretization of the Laplace equation holding for every internal point, 
$k$ being harmonic, that is: $\forall j$ and $\forall s_{i} \neq \pm \hat{s}, \pm 0$ we have

$$
\begin{aligned}
0=\frac{1}{h^{2}}\left(-4 k\left(s_{i}, \theta_{j}\right)\right. & +k\left(s_{i-1}, \theta_{j}\right)+k\left(s_{i+1}, \theta_{j}\right) \\
& \left.+k\left(s_{i}, \theta_{j-1}\right)+k\left(s_{i}, \theta_{j+1}\right)\right)
\end{aligned}
$$

Second, accounting for $k$ 's convergence to constants at $s= \pm \infty$, we set Neuman boundary conditions at the external boundaries $s_{i}= \pm \hat{s}$

$$
\begin{array}{ll}
k\left(-\hat{s}, \theta_{j}\right)=k\left(-\hat{s}+h, \theta_{j}\right) & \forall j \\
k\left(\hat{s}, \theta_{j}\right)=k\left(\hat{s}-h, \theta_{j}\right) & \forall j
\end{array}
$$

Third, we have the $k$-value, and $k$-derivative pair of welding equations from Eq. 15, between the internal boundaries $s=-0$, associated with $k_{-}$, and $s=+0$ associated with $k_{+}$.

For every $j$ we will associate one such pair of equations with every value $k\left(-0, \theta_{j}\right)$, and similarly with every value $k\left(+0, \theta_{j}\right)$. We separate the equations for $k_{-}$from those for $k_{+}$because the values of $\Psi\left(\theta_{j}\right)$ involved in the equation for $k\left(-0, \theta_{j}\right)$ do not necessarily fall on some grid line $\theta_{\tilde{j}}$, since $\Psi$ is a continuous welding diffeomorphism that does not typically send $\theta_{j}$ into some other grid line $\theta_{j}$. (Symmetrically, when focusing on the pair of welding equations for $k\left(+0, \theta_{j}\right)$ we may have that $\Psi^{-1}\left(\theta_{j}\right)$ is not a grid line.)

For every grid line $\theta_{j}$ we use the following second-order discretizations for $\frac{\partial k \pm}{\partial s}$

$$
\begin{aligned}
\left.\frac{\partial k}{\partial s}\right|_{(-0, \theta)}= & \frac{3}{2 h} k(-0, \theta)-\frac{2}{h} k(-0-h, \theta)+ \\
& \frac{1}{2 h} k(-0-2 h, \theta)+O\left(h^{2}\right)
\end{aligned}
$$

and

$$
\begin{aligned}
\left.\frac{\partial k}{\partial s}\right|_{(+0, \Psi)=} & -\left.\frac{3}{2 h} k_{+}\right|_{(+0, \Psi)}+\left.\frac{2}{h} k\right|_{(+0+h, \Psi)}- \\
& \left.\frac{1}{2 h} k\right|_{(+0+2 h, \Psi)}+O\left(h^{2}\right) .
\end{aligned}
$$

To replace the first term on the right, $\left.k\right|_{\left(+0, \Psi\left(\theta_{j}\right)\right)}$, we may simply use the value of $k$ at the grid point $\left(-0, \theta_{j}\right)$, via the $k$-value welding equation from Eq. 15

$$
\left.k_{+}\right|_{\left(+0, \Psi\left(\theta_{j}\right)\right)}=k\left(-0, \theta_{j}\right)-\Psi\left(\theta_{j}\right)+\theta_{j} .
$$

The other two values of $k$ participating in Eq. 20 may each be simply interpolated from the nearest three grid points along the $\theta$-direction. We use three such values to keep an approximation of order $h^{2}$. More precisely, for every $s$-column, and specifically for $s=+0+h$ and $s=+0+2 h$, we can write the exact interpolation relations

$$
\begin{aligned}
& \left.k\right|_{(s, \Psi)}=\left.\frac{\left(\Psi-\theta_{j_{2}}\right)\left(\Psi-\theta_{j_{3}}\right)}{\left(\theta_{j_{1}}-\theta_{j_{2}}\right)\left(\theta_{j_{1}}-\theta_{j_{3}}\right)} k\right|_{\left(s, \theta_{j_{1}}\right)}+O\left(h^{2}\right)+ \\
& \left.\frac{\left(\Psi-\theta_{j_{1}}\right)\left(\Psi-\theta_{j_{3}}\right)}{\left(\theta_{j_{2}}-\theta_{j_{1}}\right)\left(\theta_{j_{2}}-\theta_{j_{3}}\right)} k\right|_{\left(s, \theta_{j_{2}}\right)}+\left.\frac{\left(\Psi-\theta_{j_{1}}\right)\left(\Psi-\theta_{j_{2}}\right)}{\left(\theta_{j_{3}}-\theta_{j_{1}}\right)\left(\theta_{j_{3}}-\theta_{j_{2}}\right)} k\right|_{\left(s, \theta_{j_{3}}\right)},
\end{aligned}
$$

where $\theta_{j_{1}}, \theta_{j_{2}}$ and $\theta_{j_{3}}$ are the closest grid points to $\Psi$.

Substituting Eq. 21 and Eq. 22 in Eq. 20 we get from the last equation in Eq. 15 an equation between exactly 9 grid values. We associate this equation with the unknown $k\left(-0, \theta_{j}\right)$. A similar equation is associated with $k\left(+0, \theta_{j}\right)$ for every $\theta_{j}$. Together with Eq. 17 and Eq. 18 we have thus associated one equation with every grid point $\left(s_{i}, \theta_{j}\right)$. See Fig. 3 for exemplifying the three different types of equations.

Notice however that the solution is still not uniquely fixed. Adding a constant to any solution of this system will keep it a solution still. Thus the system is singular. So we first need to add one more equation that will determine that constant. Recalling that $k \rightarrow 0$ as $s \rightarrow \infty$ (cf. Eq. 11), a natural numerical equivalent condition would be that $\int_{\theta=0}^{2 \pi} k(\infty, \theta)=0$, and in its descretized form

$$
h \sum_{j} k\left(\hat{s}, \theta_{j}\right)=0 .
$$

(We could in principle set a one grid-point value of $k$ but this is less favorable numerically in general, and somewhat less amendable when carrying the set of differential equations in Eq. 15 to other, coarser grids.)

We now have one equation more than variables. Up to round-off errors the system has a unique solution since the equations are dependent. But for the numerical solver to work properly we add another unknown, say $\epsilon$, to some of the equations making the new system non-singular. Since the system without this addition has a unique solution $\epsilon$ will actually turn out to be zero up to round-off errors. We have chosen to add $\epsilon$ to each of the $k$-derivative welding equations, although other choices could be made as well.

Having computed the values of $k$ over the $k$-grid we note that in order to get the resulting shape $\Gamma$ we only need the values of $g(s, \theta)$ and hence of $h(s, \theta)$ at either one of the internal boundaries $s= \pm 0$. We can use a discretized version of the first Cauchy-Riemann equation presented in Eq. 13 in order to approximate $\frac{\partial h}{\partial \theta}$ on $s= \pm 0$, at exactly midpoints between the $k$-grid points. Specifically we write

$$
\begin{aligned}
& \frac{h\left(-0, \theta_{j+1}\right)-h\left(-0, \theta_{j}\right)}{h}= \\
& -\frac{1}{2}\left(\left.\frac{\partial k}{\partial s}\right|_{\left(-0, \theta_{j+1}\right)}+\left.\frac{\partial k}{\partial s}\right|_{\left(-0, \theta_{j}\right)}\right)+O\left(h^{2}\right),
\end{aligned}
$$

where $\left.\frac{\partial k}{\partial s}\right|_{\left(-0, \theta_{j+1}\right)}$ and $\left.\frac{\partial k}{\partial s}\right|_{\left(-0, \theta_{j}\right)}$ were already computed during the process of computing $k$, via Eq. 19. We can easily integrate the values $\left\{h\left(-0, \theta_{j}\right)\right\}_{j}$ out of their differences computed in Eq. 24, up to a global additive constant that does not matter in terms of the resulting $\Gamma$.

From $\left\{k\left(-0, \theta_{j}\right)\right\}_{j}$ and $\left\{h\left(-0, \theta_{j}\right)\right\}_{j}$ we have $\left\{g\left(-0, \theta_{j}\right)\right\}_{j}$ via Eq. 10, and can get $\left\{f\left(-0, \theta_{j}\right)\right\}_{j}$ via Eq. 5, and eventually $\Gamma$. 


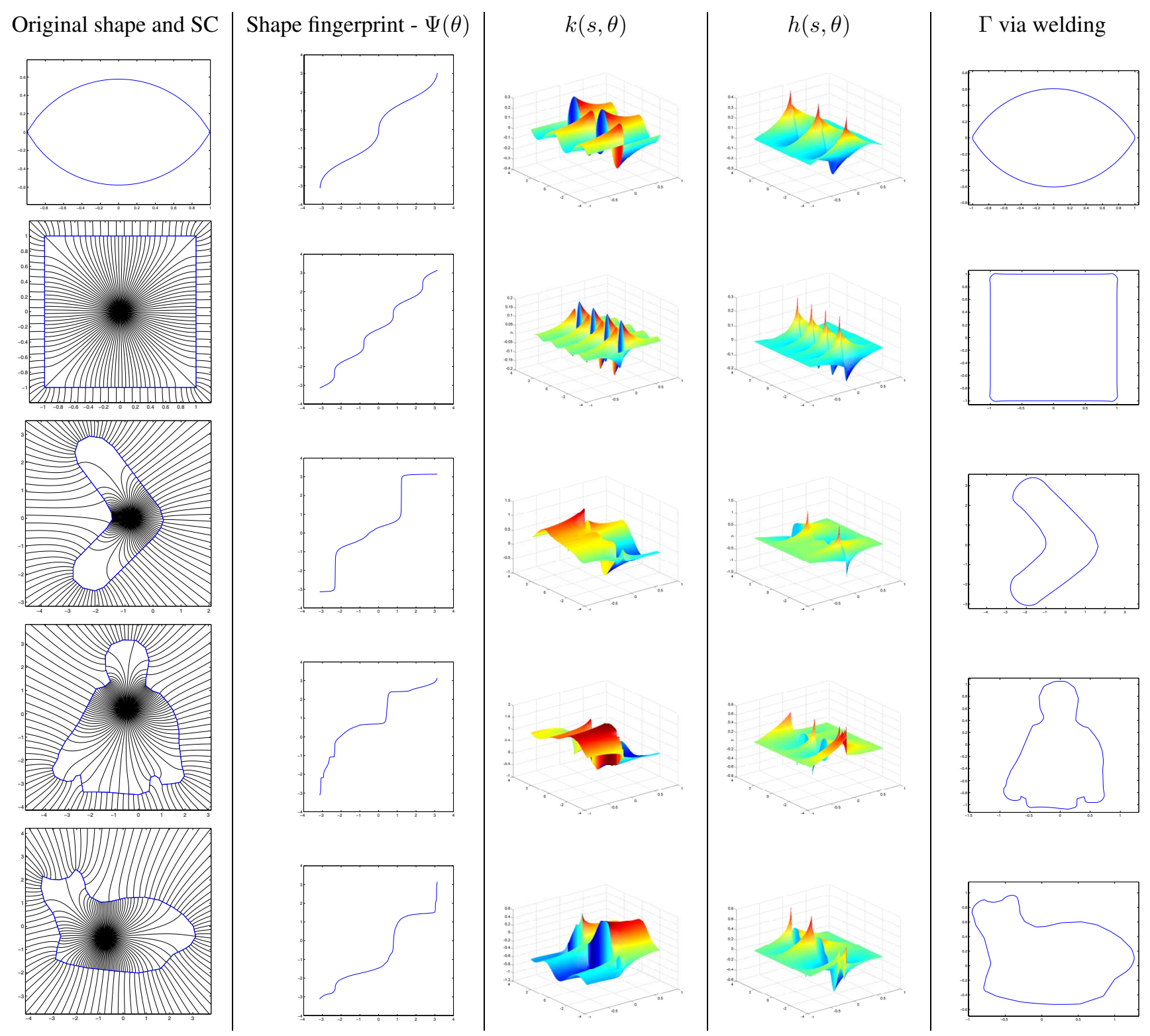

Figure 4: Experimental results. For each shape we present a row of five columns. (From left) 1st column: The conformal mappings $\Phi_{-}$and $\Phi_{+}$ carrying the two copies of the unit disc, $\Delta_{-}$and $\Delta_{+}$onto the interior and the exterior of the $\Gamma$ shape, as explained in Sec. 2.1. The figure illustrates how a homogenous radial grid on $\Delta_{-}$and $\Delta_{+}$, made of concentric lines through the origin, is mapped differently into the interior and exterior of the $\Gamma$ shape. Note the differences in the densities of the radial grid lines, along $\Gamma$, between the interior and the exterior maps. This difference in densities along $\Gamma$ is exactly what is encoded by the diffeomorphisms $\Psi \in G / H$ that match $\Gamma$, and is the fingerprint of the shape. 2nd column: Shape fingerprint $\Psi(\theta):[0,2 \pi] \rightarrow[0,2 \pi]$, computed analytically for the first row, and numerically via Schwarz-Christoffel for the last four rows. 3rd column: $k(s, \theta)$, computed over the grid $\left(s_{i}, \theta_{j}\right)$. 4th column: $h(s, \theta)$, conjugated from $k(s, \theta)$ over the grid $\left(s_{i}, \theta_{j}\right)$. 5th column: the shape $\Gamma$, as it results from $\Psi$ via welding, up to scale and translation, obtained by drawing $f(s=0)$ in the complex plane.

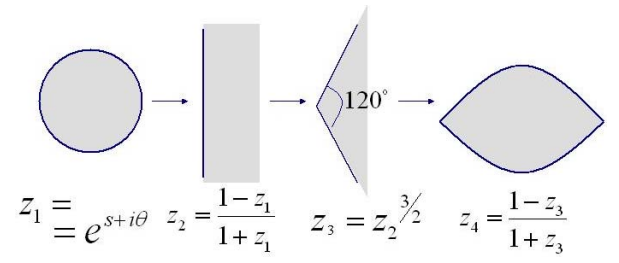

Figure 5: Example: The construction of $\Phi_{-}-$the conformal mapping of the interior of the unit disc onto the interior of the "eye" shape, presented in steps. 


\section{Results and Examples}

We implemented a solver for the set of welding equations described in Eq. 15, according to Sec. 4.2.2. To go back and forth between $\overline{\mathcal{S}}$ and $G / H$ we start with a shape $\Gamma \in \overline{\mathcal{S}}$, and using the Schwarz-Christoffel transformation (Sec. 4.1) we compute the two conformal mappings, $\Phi_{-}$and $\Phi_{+}$of the unit disc to the interior and exterior of the shape, correspondingly as explained in Sec. 2.1. We may then obtain a diffeomorphism $\Psi$ from the coset in $G / H$ describing $\Gamma$ by defining $\left.\Psi \doteq \Phi_{+}{ }^{-1} \circ \Phi_{-}\right|_{S^{1}}$. To go back from $\Psi$ to $\Gamma$ we follow Sec. 4.2.2 for welding in order to get $f$, and demonstrate that the resulting $\Gamma$ is indeed the one we started with.

The first example we solve for represents a family of shapes for which the conformal mappings $\Phi_{-}$and $\Phi_{+}$can be solved analytically. Following the construction of $\Phi_{-}$ for the "eye" shape (Fig. 5) we can analytically obtain that $\Psi(\theta)=\Phi_{+}^{-1}\left(\Phi_{-}\left(e^{i \theta}\right)\right)=2 \operatorname{atan}\left(\operatorname{sign} \theta \tan ^{\frac{1}{2}}\left(\frac{|\theta|}{2}\right)\right)$ for $\theta \in[-\pi, \pi]$. The eye shape, $\Psi(\theta)$ and the results of solving Eq. 15 for that $\Psi$, as described in Sec. 4.2.2, are presented in the first row of Fig. 5. The fact that the $\Gamma$ obtained by welding (right) is identical, up to scale and translation, to the original shape (left) demonstrates the successful reconstruction of $\Gamma$ from $\Psi$, via welding.

We continue by experimenting with four additional, more complex shapes, a square, a boomerang, a person's upper-body and a cat (see Fig. 5). For these we apply the Schwarz-Christoffel transformation in order to obtain $\Psi(\theta)$. Hence $\Psi(\theta), \Psi^{\prime}(\theta)$ and $\Psi^{-1}(\theta)$ involved in Eq. 15 are computed numerically. Note again the striking similarity of the computed $\Gamma$ (right) to the original shape (left). Recall from Eq. 16 the way $k$ and $h$ (Fig. 4, columns 3-4) describe $\Gamma$. In our current straightforward implementation we are limited in the size of the $(s, \theta)$-grid we can solve for. This results in the minor distortions in $k, h$ and the resulting $\Gamma$. We are currently implementing a multigrid algorithm for a faster and more accurate solution, that will alow better resolution for handling much more complex shapes.

\section{Summary and Conclusions}

We introduce a metric space-of-shapes that arises from conformal mappings, through the mathematical theory of complex analysis. In this space, it appears very likely to be true that the shortest path between each two shapes is unique, and is given by a geodesic connecting them, providing a path for morphing between them. Every shape is represented in this space by an equivalence class of "fingerprints" each of which is a diffeomorphism of the unit circle to itself. We solved the welding problem to allow moving back and forth between shapes and this space-of-shapes, thus allowing the continuation of the research of shapes within this space. Indeed, our next step will be to compute the geodesics between shapes. We expect these to reflect the appealing structure-preserving properties of conformal mappings, and to be very relevant to the comparison and classification of shapes.

\section{References}

[1] Y. Amit, U. Grenander, and M. Piccioni. Structural image restoration through deformable templates. J Am Statis Assoc, 86(414):376-387, 1991.

[2] R. Basri, L. Costa, D. Geiger, and D. Jacobs. Determining the similarity of deformable shapes. Vision Research, 38:2365-2385, 1998.

[3] S. Belongie, J. Malik, and J. Puzicha. Shape matching and object recognition using shape contexts. PAMI, 24(4):509-522, 2002.

[4] I. Biederman. Human image understanding: recent research and a theory. Computer Vision, Graphics, and Image Processing, 32:2973, 1985.

[5] R. Bolles and R. Chan. Recognizing and locating partially visible objects: the local feature-focus method. Int J Robot Res, 1(3):57-82, 1982.

[6] M. J. Bowick and A. Lahiri. The Ricci curvature of $\operatorname{Diff}\left(s^{1}\right) / \operatorname{SL}(2, \mathcal{R})$. J. of Math. Physics, 29:1979-1981, 1988. South Carolina.

[7] S. Carlsson. Order structure, correspondence and shape based categories. International Workshop on Shape, Contour and Grouping , Springer Lecture Notes in Computer Science, page 1681, 1999.

[8] T. A. Driscoll. A MATLAB toolbox for Schwarz-Christoffel mapping. ACM Trans. Math. Soft., 22:168-186, 1996.

[9] R.O. Duda, P.E. Hart, and D.G. Stork. Pattern Classification. Wiley, New York, 2 edition, 2001.

[10] P. T. Fletcher, C. Lu, and S. Joshi. Statistics of shape via principal geodesic analysis on lie groups. CVPR, pages 95-101, 2003.

[11] Y. Gdalyahu and D. Weinshall. Flexible syntactic matching of curves and its application to automatic hierarchical classification of silhouettes. PAMI, 21(12):1312-1328, 1999.

[12] D. Geiger, A. Gupta, L. Costa, and J. Vlontzos. Dynamic programming for detecting, tracking and matching deformable contours. IEEE Trans Pattern Anal Mach Intell, 17(3):294-302, 1995.

[13] U. Grenander and M. Miller. Computational anatomy. Quart. of Appl. Math., 56, 1998.

[14] X. Gu and S. T. Yau. Surface classification using conformal structures. ICCV, pages 701-708, 2003.

[15] E. Hildreth. The Measurement of Visual Motion. MIT Press, Cambridge, 1984.

[16] M. Kass, A. Witkin, and D. Terzopoulos. Snakes: Active contour models. IJCV, I(4):321-331, 1988.

[17] B. B. Kimia, A. R. Tannenbaum, and S. W. Zucker. Shapes, shocks, and deformations. IJCV, 15(3):189-224, 1995.

[18] O. Lehto. Univalent Functions and Teichmuller Spaces. SpringerVerlag New York Berlin Heidelberg, 1986.

[19] D. Mumford. Mathematical theories of shape: do they model perception? SPIE Geom Meth Comp Vis, 1570:2-10, 1991.

[20] S. Sclaroff and A. Pentland. Modal matching for correspondence and recognition. PAMI, 17(6):545-561, August 1995.

[21] T. B. Sebastian, P. N. Klein, and B. B. Kimia. Recognition of shapes by editing shock graphs. ICCV, pages 755-762, 2001.

[22] S. Ullman. Aligning pictorial descriptions: an approach to object recognition. Cognition, 32(3):193-254, 1989.

[23] A. Yuille. Deformable templates for face recognition. J. Cognitive Neuroscience, 3(1):59-71, 1991. 